\title{
Uniqueness problems on entire functions that share a small function with their difference operators
}

\section{BaoQin Chen and Sheng Li}

"Correspondence: lish_Is@sina.com College of Science, Guangdong Ocean University, Zhangjiang, 524088, P.R. China

\begin{abstract}
In this paper, we consider uniqueness problems on entire functions that share a small periodic entire function with their two difference operators and obtain some results. Our first theorem provides a difference analogue of a result of Li and Yang (J. Math. Anal. Appl. 253(1):50-57, 2001).

MSC: Primary 30D35; secondary 39B32

Keywords: uniqueness; entire functions; difference operators
\end{abstract}

\section{Introduction and main results}

Throughout this paper, a meromorphic function always means meromorphic in the whole complex plane, and $c$ always means a nonzero constant. We use the basic notations of the Nevanlinna theory of meromorphic functions such as $T(r, f), m(r, f), N(r, f)$ and $\bar{N}(r, f)$ as explained in [1-3]. In addition, we say that a meromorphic function $a(z)$ is a small function of $f(z)$ if $T(r, a)=S(r, f)$, where $S(r, f)=o(T(r, f))$, as $r \rightarrow \infty$ outside of a possible exceptional set of finite logarithmic measure.

For a meromorphic function $f(z)$, we define its shift by $f(z+c)$, and define its difference operators by

$$
\Delta_{c} f(z)=f(z+c)-f(z) \quad \text { and } \quad \Delta_{c}^{n} f(z)=\Delta_{c}^{n-1}\left(\Delta_{c} f(z)\right), \quad n \in \mathbb{N}, n \geq 2 .
$$

In particular, $\Delta_{c}^{n} f(z)=\Delta^{n} f(z)$ for the case $c=1$.

Let $f(z)$ and $g(z)$ be two meromorphic functions, and let $a(z)$ be a small function of $f(z)$ and $g(z)$. We say that $f(z)$ and $g(z)$ share $a(z)$ IM, provided that $f(z)-a(z)$ and $g(z)-a(z)$ have the same zeros ignoring multiplicities. Similarly, we say that $f(z)$ and $g(z)$ share $a(z)$ CM, provided that $f(z)-a(z)$ and $g(z)-a(z)$ have the same zeros counting multiplicities.

The problem on meromorphic functions sharing small functions with their derivatives is an important topic of uniqueness of meromorphic functions.

In 1986, Jank, Mues and Volkmann [4] proved the following result.

Theorem A ([4]) Let $f$ be a nonconstant meromorphic function, and let $a \neq \equiv$ be a finite constant. Iff, $f^{\prime}$, and $f^{\prime \prime}$ share the value a $C M$, then $f \equiv f^{\prime}$.

02014 Chen and Li; licensee Springer. This is an Open Access article distributed under the terms of the Creative Commons Attribution License (http://creativecommons.org/licenses/by/2.0), which permits unrestricted use, distribution, and reproduction in any medium, provided the original work is properly cited. 
Many authors have been considering about some related cases, and got some interesting results (see, e.g., [5, 6]). In 2001, Li and Yang [5] obtained the following result for a special case that $f(z)$ is an entire function, and $f, f^{\prime}$, and $f^{(n)}$ share one value.

Theorem B ([5]) Let $f(z)$ be an entire function, let a be a finite nonzero constant, and let $n(\geq 2)$ be a positive integer. If $f, f^{\prime}$, and $f^{(n)}$ share the value a $C M$, then $f$ assumes the form

$$
f(z)=b e^{c z}-\frac{a(1-c)}{c}
$$

where $b, c$ are nonzero constants and $c^{n-1}=1$.

Recently, a number of papers (including [7-12]) have focused on difference analogues of Nevanlinna theory. In addition, many papers have been devoted to the investigation of the uniqueness problems related to meromorphic functions and their shifts or their difference operators and got a lot of results (see, e.g., [13-15]).

Our aim in this paper is to investigate uniqueness problems on entire functions that share a small periodic entire function with their two difference operators and provide a difference analogue of Theorem B. We now state the following theorem, which is the main result of this paper.

Theorem 1.1 Let $f(z)$ be a nonconstant entire function of finite order, and let a $(z)(\not \equiv 0) \in$ $S(f)$ be a periodic entire function with period c. If $f(z), \Delta_{c} f$, and $\Delta_{c}^{n} f(n \geq 2)$ share $a(z) C M$, then $\Delta_{c}^{n} f \equiv \Delta_{c} f$.

\section{Examples}

(1) Let $f(z)=e^{\left(\frac{\pi}{4} i+\ln \sqrt{2}\right) z}+1+i$, then $\Delta f \equiv \Delta^{5} f=i e^{\left(\frac{\pi}{4} i+\ln \sqrt{2}\right) z}$, and hence $f(z), \Delta f$, and $\Delta^{5} f$ share $1 \mathrm{CM}$, but $f(z) \not \equiv \Delta f$. This example shows that the conclusion $\Delta_{c}^{n} f \equiv \Delta_{c} f$ in Theorem 1.1 cannot be extended to $f(z) \equiv \Delta_{c} f$ in general.

(2) Let $f(z)=e^{z \ln 3}$, then $\Delta f \equiv 2 f(z), \Delta^{n} f \equiv 2^{n} f(z)$, and hence $f(z), \Delta f$ and $\Delta^{n} f$ share 0 $\mathrm{CM}$, but $\Delta^{n} f \equiv 2^{n-1} \Delta f \not \equiv \Delta(n \geq 2)$. This example shows that the restriction $a(z) \not \equiv 0$ in Theorem 1.1 is necessary.

Remark In the above example (1), $f(z)=e^{\left(\frac{\pi}{4} i+\ln \sqrt{2}\right) z}+1+i$ can be changed to $f(z)=$ $g(z) e^{\left(\frac{\pi}{4} i+\ln \sqrt{2}\right) z}+1+i$, where $g(z)$ is a periodic entire function with period 1 , and the result still holds. This shows that the order of the function $f(z)$ in Theorem 1.1 is not always one.

As a continuation of Theorem 1.1 and example (2) above, we prove the following result.

Theorem 1.2 Let $f(z)$ be a nonconstant entire function of finite order. Iff $(z), \Delta_{c} f$, and $\Delta_{c}^{n} f$ $(n \geq 2)$ share $0 C M$, then $\Delta_{c}^{n} f \equiv C \Delta_{c} f$, where $C$ is a nonzero constant.

\section{Proof of Theorem 1.1}

Firstly, we present some lemmas which will be needed in the proof of Theorem 1.1.

Lemma 2.1 ([11]) Let $c \in \mathbb{C}, n \in \mathbb{N}$, and let $f(z)$ be a meromorphic function of finite order. Then for any small periodic function a $(z)$ with period $c$, with respect to $f(z)$, 


$$
m\left(r, \frac{\Delta_{c}^{n} f}{f-a}\right)=S(r, f)
$$

where the exceptional set associated with $S(r, f)$ is of at most finite logarithmic measure.

Lemma 2.2 ([3]) Suppose that $f_{j}(z)(j=1,2, \ldots, n)$ and $g_{j}(z)(j=1,2, \ldots, n)(n \geq 2)$ are entire functions satisfying

(i) $\sum_{j=1}^{n} f_{j}(z) e^{g_{j}(z)} \equiv 0$;

(ii) the orders of $f_{j}$ are less than that of $e^{g_{h}(z)-g_{k}(z)}$ for $1 \leq j \leq n, 1 \leq h<k \leq n$,

then $f_{j}(z) \equiv 0(j=1,2, \ldots, n)$.

Proof of Theorem 1.1 Suppose on the contrary to the assertion that $\Delta_{c}^{n} f \not \equiv \Delta_{c} f$. Note that $f(z)$ is a nonconstant entire function of finite order. By Lemma 2.1, for $n \geq 2$, we have

$$
T\left(r, \Delta_{c}^{n} f\right)=m\left(r, \Delta_{c}^{n} f\right) \leq m\left(r, \frac{\Delta_{c}^{n} f}{f(z)}\right)+m(r, f(z)) \leq T(r, f(z))+S(r, f) .
$$

Similarly,

$$
T\left(r, \Delta_{c} f\right) \leq T(r, f(z))+S(r, f) .
$$

Since $f(z), \Delta_{c} f$, and $\Delta_{c}^{n} f$ share $a(z) \mathrm{CM}$, we have

$$
\frac{\Delta_{c}^{n} f-a(z)}{f(z)-a(z)}=e^{\alpha(z)}, \quad \frac{\Delta_{c} f-a(z)}{f(z)-a(z)}=e^{\beta(z)},
$$

where $\alpha(z)$ and $\beta(z)$ are polynomials.

Set

$$
\varphi(z)=\frac{\Delta_{c}^{n} f-\Delta_{c} f}{f(z)-a(z)} .
$$

From (2.1) and (2.2), we get $\varphi(z)=e^{\alpha(z)}-e^{\beta(z)}$. Then by supposition and (2.1), we see that $\varphi(z) \not \equiv 0$. By Lemma 2.1, we deduce that

$$
\begin{aligned}
T(r, \varphi) & =m(r, \varphi) \\
& \leq m\left(r, \frac{\Delta_{c}^{n} f}{f(z)-a(z)}\right)+m\left(r, \frac{\Delta_{c} f}{f(z)-a(z)}\right)+S(r, f)=S(r, f) .
\end{aligned}
$$

Note that $\frac{e^{\alpha}}{\varphi}-\frac{e^{\beta}}{\varphi}=1$. By using the second main theorem and (2.3), we have

$$
\begin{aligned}
T\left(r, \frac{e^{\alpha}}{\varphi}\right) & \leq \bar{N}\left(r, \frac{e^{\alpha}}{\varphi}\right)+\bar{N}\left(r, \frac{\varphi}{e^{\alpha}}\right)+\bar{N}\left(r, \frac{1}{e^{\alpha} / \varphi-1}\right)+S\left(r, \frac{e^{\alpha}}{\varphi}\right) \\
& =\bar{N}\left(r, \frac{e^{\alpha}}{\varphi}\right)+\bar{N}\left(r, \frac{\varphi}{e^{\alpha}}\right)+\bar{N}\left(r, \frac{\varphi}{e^{\beta}}\right)+S\left(r, \frac{e^{\alpha}}{\varphi}\right) \\
& =S(r, f)+S\left(r, \frac{e^{\alpha}}{\varphi}\right) .
\end{aligned}
$$

Thus, by (2.3) and (2.4), we have $T\left(r, e^{\alpha}\right)=S(r, f)$. Similarly, $T\left(r, e^{\beta}\right)=S(r, f)$. 
Now we divide this proof into the following two steps.

Step 1. Suppose that $\beta(z)$ is not a constant. Now we rewrite the second equation in (2.1) as

$$
\Delta_{c} f=a_{1}(z) f(z)+b_{1}(z)
$$

and

$$
f(z+c)=a_{0}(z) f(z)+b_{0}(z)
$$

where $a_{0}(z)=e^{\beta(z)}+1, a_{1}(z)=e^{\beta(z)}, b_{0}(z)=b_{1}(z)=a(z)\left(1-e^{\beta(z)}\right)$.

We deduce that

$$
\begin{aligned}
\Delta_{c}^{2} f= & \Delta_{c} f(z+c)-\Delta_{c} f(z) \\
= & a_{1}(z+c) f(z+c)+b_{1}(z+c)-a_{1}(z) f(z)-b_{1}(z) \\
= & e^{\beta(z+c)} f(z+c)+a(z+c)\left(1-e^{\beta(z+c)}\right)-e^{\beta(z)} f(z)-a(z)\left(1-e^{\beta(z)}\right) \\
= & e^{\beta(z+c)}\left[\left(e^{\beta(z)}+1\right) f(z)+a(z)\left(1-e^{\beta(z)}\right)\right]+a(z)\left(1-e^{\beta(z+c)}\right) \\
& -e^{\beta(z)} f(z)-a(z)\left(1-e^{\beta(z)}\right) \\
= & \left(e^{\beta(z+c)+\beta(z)}+e^{\beta(z+c)}-e^{\beta(z)}\right) f(z)+a(z)\left(e^{\beta(z)}-e^{\beta(z+c)+\beta(z)}\right), \\
\Delta_{c}^{3} f= & \Delta_{c}^{2} f(z+c)-\Delta_{c}^{2} f(z) \\
= & \left(e^{\beta(z+2 c)+\beta(z+c)}+e^{\beta(z+2 c)}-e^{\beta(z+c)}\right) f(z+c)+a(z+c)\left(e^{\beta(z+c)}-e^{\beta(z+2 c)+\beta(z+c)}\right) \\
& -\left(e^{\beta(z+c)+\beta(z)}+e^{\beta(z+c)}-e^{\beta(z)}\right) f(z)-a(z)\left(e^{\beta(z)}-e^{\beta(z+c)+\beta(z)}\right) \\
= & \left(e^{\beta(z+2 c)+\beta(z+c)}+e^{\beta(z+2 c)}-e^{\beta(z+c)}\right)\left[\left(e^{\beta(z)}+1\right) f(z)+a(z)\left(1-e^{\beta(z)}\right)\right] \\
& +a(z)\left(e^{\beta(z+c)}-e^{\beta(z+2 c)+\beta(z+c)}\right)-\left(e^{\beta(z+c)+\beta(z)}+e^{\beta(z+c)}-e^{\beta(z)}\right) f(z) \\
& -a(z)\left(e^{\beta(z)}-e^{\beta(z+c)+\beta(z)}\right) \\
= & \left(e^{\beta(z+2 c)+\beta(z+c)+\beta(z)}+e^{\beta(z+2 c)+\beta(z+c)}+e^{\beta(z+2 c)+\beta(z)}-2 e^{\beta(z+c)+\beta(z)}\right. \\
& \left.+e^{\beta(z+2 c)}-2 e^{\beta(z+c)}+e^{\beta(z)}\right) f(z)+a(z)\left(-e^{\beta(z+2 c)+\beta(z+c)+\beta(z)}\right. \\
& \left.-e^{\beta(z+2 c)+\beta(z)}+2 e^{\beta(z+c)+\beta(z)}+e^{\beta(z+2 c)}-e^{\beta(z)}\right) .
\end{aligned}
$$

That is,

$$
\Delta_{c}^{2} f=a_{2}(z) f(z)+b_{2}(z), \quad \Delta_{c}^{3} f=a_{3}(z) f(z)+b_{3}(z),
$$

where

$$
\begin{aligned}
a_{2}(z)= & e^{\beta(z+c)+\beta(z)}+e^{\beta(z+c)}-e^{\beta(z)}, \\
a_{3}(z)= & e^{\beta(z+2 c)+\beta(z+c)+\beta(z)}+e^{\beta(z+2 c)+\beta(z+c)}+e^{\beta(z+2 c)+\beta(z)} \\
& -2 e^{\beta(z+c)+\beta(z)}+e^{\beta(z+2 c)}-2 e^{\beta(z+c)}+e^{\beta(z)}, \\
b_{2}(z)= & a(z)\left(e^{\beta(z)}-e^{\beta(z+c)+\beta(z)}\right), \\
b_{3}(z)= & a(z)\left(-e^{\beta(z+2 c)+\beta(z+c)+\beta(z)}-e^{\beta(z+2 c)+\beta(z)}+2 e^{\beta(z+c)+\beta(z)}+e^{\beta(z+2 c)}-e^{\beta(z)}\right) .
\end{aligned}
$$


Let $\Omega=\{0,1, \ldots, n-1\}$ be a finite set of $n$ elements, and denote $P(\Omega)=\{\varnothing,\{0\},\{1\}, \ldots,\{n-$ $1\},\{0,1\},\{0,2\}, \ldots, \Omega\}$, where $\varnothing$ is an empty set. Then by an argument similar to the above, we deduce that

$$
\Delta_{c}^{n} f=a_{n}(z) f(z)+b_{n}(z)
$$

with

$$
\begin{aligned}
a_{n}(z) & =\sum_{A \in P(\Omega) \backslash\{\varnothing\}} \lambda_{A} \prod_{j \in A} e^{\beta(z+j c)} \\
& =\lambda_{n, 0} \prod_{j=0}^{n-1} e^{\beta(z+j c)}+\sum_{t=0}^{n-1} \lambda_{n-1, t} \prod_{j=0, j \neq t}^{n-1} e^{\beta(z+j c)}+\cdots+\sum_{t=0}^{n-1} \lambda_{1, t} e^{\beta(z+t c)}, \\
b_{n}(z) & =a(z) Q_{n}\left(e^{\beta(z)}\right),
\end{aligned}
$$

where $A$ is any element of $P(\Omega), \lambda_{A}$, and $\lambda_{s, t}$, for $s=1,2 \ldots, n, t=0,1, \ldots, C_{n}^{s}-1\left(C_{n}^{s}=\frac{n !}{s !(n-s) !}\right)$ are nonzero constants. In particular, $\lambda_{n, 0}=1$, and

$$
\sum_{t=0}^{n-1} \lambda_{1, t} e^{\beta(z+t c)}=\Delta_{c}^{n-1} e^{\beta(z)}
$$

Moreover, $Q_{n}\left(e^{\beta(z)}\right)$ is a polynomial of $e^{\beta(z)}$ and its shifts $e^{\beta(z+c)}, e^{\beta(z+2 c)}, \ldots, e^{\beta(z+(n-1) c)}$.

Now set $\beta(z)=l_{m} z^{m}+l_{m-1} z^{m-1}+\cdots+l_{0}$, where $l_{m}, \ldots, l_{0}$ are constants satisfying $l_{m} \neq 0$ and $m \geq 1$. Obviously, for $j=0,1, \ldots, n-1$, we have

$$
\beta(z+j c)=l_{m} z^{m}+\left(l_{m-1}+m l_{m} j c\right) z^{m-1}+\cdots+\sum_{k=0}^{m} l_{k} j^{k} c^{k}
$$

By the above equation and (2.9), we obtain

$$
\begin{aligned}
a_{n}(z)= & e^{n l_{m} z^{m}+P_{n, 0}(z)}+\lambda_{n-1,0} e^{(n-1) l_{m} z^{m}+P_{n-1,0}(z)}+\cdots \\
& +\lambda_{n-1, n-1} e^{(n-1) l_{m} z^{m}+P_{n-1, n-1}(z)}+\cdots+\lambda_{1,0} e^{l_{m} z^{m}+P_{1,0}(z)}+\cdots \\
& +\lambda_{1, n-1} e^{l_{m} z^{m}+P_{1, n-1}(z)} .
\end{aligned}
$$

Here $P_{s, t}(z)$, for $s=1,2, \ldots, n, t=0,1, \ldots, C_{n}^{s}-1$, are polynomials with degree less than $m$. Rewrite the first equation in (2.1) as

$$
\Delta_{c}^{n} f-e^{\alpha(z)} f(z)=a(z)\left(1-e^{\alpha(z)}\right)
$$

This together with (2.8) gives

$$
\left(a_{n}(z)-e^{\alpha(z)}\right) f(z)=a(z)\left(1-e^{\alpha(z)}\right)-b_{n}(z)
$$


Notice that $a(z) \in S(f), T\left(r, e^{\alpha}\right)=S(r, f)$, and $T\left(r, e^{\beta}\right)=S(r, f)$. If $a_{n}(z)-e^{\alpha(z)} \not \equiv 0,(2.12)$ yields

$$
\begin{aligned}
T(r, f)+S(r, f) & =T\left(r,\left(a_{n}(z)-e^{\alpha(z)}\right) f(z)\right) \\
& =T\left(r, a(z)\left(1-e^{\alpha(z)}\right)-b_{n}(z)\right)=S(r, f) .
\end{aligned}
$$

That is impossible.

Hence $a_{n}(z)-e^{\alpha(z)} \equiv 0$. This together with (2.11) gives

$$
\begin{aligned}
& e^{P_{n, 0}(z)} e^{n l_{m} z^{m}}+\left(\lambda_{n-1,0} e^{P_{n-1,0}(z)}+\cdots+\lambda_{n-1, n-1} e^{P_{n-1, n-1}(z)}\right) \\
& \cdot e^{(n-1) l_{m} z^{m}}+\cdots+\left(\lambda_{1,0} e^{P_{1,0}(z)}+\cdots+\lambda_{1, n-1} e^{P_{1, n-1}(z)}\right) e^{l_{m} z^{m}} \\
& -e^{\alpha(z)} \equiv 0 .
\end{aligned}
$$

Now we distinguish three cases as follows:

Case (i). Suppose that $\operatorname{deg} \alpha(z)>m$. Then, for any $1 \leq j \leq n$, we see that

$$
\rho\left(e^{\alpha(z)-j l_{m} z^{m}}\right)=\rho\left(e^{\alpha(z)}\right)=\operatorname{deg} \alpha(z)>m
$$

and for $1 \leq h<k \leq n$, we have

$$
\rho\left(e^{h l_{m} z^{m}-k l_{m} z^{m}}\right)=m
$$

Since $P_{s, t}(z)$, for $s=1,2 \ldots, n, t=0,1, \ldots, C_{n}^{s}-1$, are polynomials with degree less than $m$, it is easy to see that, for $s=1,2, \ldots, n-1$,

$$
\rho\left(\sum_{t=0}^{C_{n}^{s}-1} \lambda_{s, t} e^{P_{s, t}(z)}\right) \leq m-1, \quad \rho\left(e^{P_{n, 0}(z)}\right) \leq m-1
$$

By Lemma 2.2, we have $e^{P_{n, 0}(z)} \equiv 0$, which is impossible.

Case (ii). Suppose that $\operatorname{deg} \alpha(z)<m$. Then, by a similar argument to above, we can also get a contradiction.

Case (iii). Now suppose that $\operatorname{deg} \alpha(z)=m$. Set $\alpha(z)=d z^{m}+P^{*}(z)$, with $d \neq 0$ and $\operatorname{deg} P^{*}(z)<m$. Rewrite (2.13) as

$$
\begin{aligned}
& e^{P_{n, 0}(z)} e^{n l_{m} z^{m}}+\left(\lambda_{n-1,0} e^{P_{n-1,0}(z)}+\cdots+\lambda_{n-1, n-1} e^{P_{n-1, n-1}(z)}\right) \\
& \cdot e^{(n-1) l_{m} z^{m}}+\cdots+\left(\lambda_{1,0} e^{P_{1,0}(z)}+\cdots+\lambda_{1, n-1} e^{P_{1, n-1}(z)}\right) e^{l_{m} z^{m}} \\
& -e^{P^{*}(z)} e^{d z^{m}} \equiv 0 .
\end{aligned}
$$

Subcase (i). If $d \neq j l_{m}$, for any $j=1,2, \ldots, n$, then we have

$$
\rho\left(e^{d z^{m}-j l_{m} z^{m}}\right)=\rho\left(e^{\left(d-j l_{m}\right) z^{m}}\right)=m, \quad \rho\left(e^{P^{*}(z)}\right) \leq m-1 .
$$

By this together with (2.14), (2.15), (2.16), and Lemma 2.2, we can get a contradiction. 
Subcase (ii). If $d=j l_{m}$, for some $j=1,2, \ldots, n-1$. Without loss of generality, we assume that $j=n-1$. Then we rewrite $(2.16)$ as

$$
\begin{aligned}
& e^{P_{n, 0}(z)} e^{n l_{m} z^{m}}+\left(\lambda_{n-1,0} e^{P_{n-1,0}(z)}+\cdots+\lambda_{n-1, n-1} e^{P_{n-1, n-1}(z)}-e^{P^{*}(z)}\right) \\
& \cdot e^{(n-1) l_{m} z^{m}}+\cdots+\left(\lambda_{1,0} e^{P_{1,0}(z)}+\cdots+\lambda_{1, n-1} e^{P_{1, n-1}(z)}\right) e^{l_{m} z^{m}} \equiv 0 .
\end{aligned}
$$

By a similar method as the above, we can also get $e^{P_{n, 0}(z)} \equiv 0$. That is impossible.

Subcase (iii). If $d=n l_{m}$, then we rewrite (2.16) as

$$
\begin{gathered}
\left(e^{P_{n, 0}(z)}-e^{P^{*}(z)}\right) e^{n l_{m} z^{m}}+\left(\lambda_{n-1,0} e^{P_{n-1,0}(z)}+\cdots+\lambda_{n-1, n-1} e^{P_{n-1, n-1}(z)}\right) \\
\cdot e^{(n-1) l_{m} z^{m}}+\cdots+\left(\lambda_{1,0} e^{P_{1,0}(z)}+\cdots+\lambda_{1, n-1} e^{P_{1, n-1}(z)}\right) e^{l_{m} z^{m}} \equiv 0
\end{gathered}
$$

By a similar argument to the above and Lemma 2.2, we can get

$$
\lambda_{1,0} e^{P_{1,0}(z)}+\cdots+\lambda_{1, n-1} e^{P_{1, n-1}(z)} \equiv 0,
$$

which implies

$$
\left(\lambda_{1,0} e^{P_{1,0}(z)}+\cdots+\lambda_{1, n-1} e^{P_{1, n-1}(z)}\right) e^{l_{m} z^{m}} \equiv 0 .
$$

By (2.10) and (2.11), we get

$$
\Delta_{c}^{n-1} e^{\beta(z)}=\sum_{j=0}^{n-1}\left(\begin{array}{c}
n-1 \\
j
\end{array}\right)(-1)^{n-1-j} e^{\beta(z+j c)} \equiv 0 .
$$

Suppose that $m>1$. Note that for $j=0,1, \ldots, n-1$, we have

$$
\beta(z+j c)=l_{m} z^{m}+\left(l_{m-1}+m l_{m} j c\right) z^{m-1}+Q_{j}(z)
$$

where $Q_{j}(z)$ are polynomials with degree less than $m-1$.

Rewrite (2.19) as

$$
\begin{aligned}
e^{Q_{n-1}(z)} & e^{l_{m} z^{m}+\left(l_{m-1}+m l_{m}(n-1) c\right) z^{m-1}}-(n-1) e^{Q_{n-2}(z)} \\
& \cdot e^{l_{m} z^{m}+\left(l_{m-1}+m l_{m}(n-2) c\right) z^{m-1}}+\cdots+(-1)^{n-1} e^{Q_{0}(z)} e^{l_{m} z^{m}+l_{m-1} z^{m-1}} \equiv 0 .
\end{aligned}
$$

For any $0 \leq h<k \leq n-1$, we have

$$
\rho\left(e^{l_{m} z^{m}+\left(l_{m-1}+m l_{m} h c\right) z^{m-1}-\left(l_{m} z^{m}+\left(l_{m-1}+m l_{m} k c\right) z^{m-1}\right)}\right)=\rho\left(e^{m l_{m}(h-k) c z^{m-1}}\right)=m-1,
$$

and for $j=0,1, \ldots, n-1$, we see that

$$
\rho\left(e^{Q_{j}(z)}\right) \leq m-2 \text {. }
$$

By this, together with (2.20) and Lemma 2.2, we obtain $e^{Q_{n-1}(z)} \equiv 0$, which is impossible. 
Suppose that $m=1$, then $\beta(z)=l_{1} z+l_{0}$, with $l_{1} \neq 0$. It is easy to see that

$$
\begin{aligned}
& \Delta_{c} e^{\beta(z)}=e^{\beta(z+c)}-e^{\beta(z)}=e^{l_{1} z+l_{1} c+l_{0}}-e^{l_{1} z+l_{0}}=\left(e^{l_{1} c}-1\right) e^{\beta(z)}, \\
& \Delta_{c}^{2} e^{\beta(z)}=\left(e^{l_{1} c}-1\right) \Delta_{c} e^{\beta(z)}=\left(e^{l_{1} c}-1\right)^{2} e^{\beta(z)} .
\end{aligned}
$$

By induction,

$$
\Delta_{c}^{n-1} e^{\beta(z)}=\left(e^{l_{1} c}-1\right)^{n-1} e^{\beta(z)} .
$$

This together with (2.19) gives

$$
\left(e^{l_{1} c}-1\right)^{n-1} \equiv 0
$$

which yields $e^{l_{1} c} \equiv 1$. Therefore, for any $j \in \mathbb{Z}$,

$$
e^{\beta(z+j c)}=e^{l_{1} z+j l_{1} c+l_{0}}=e^{l_{1} z+l_{0}}\left(e^{l_{1} c}\right)^{j}=e^{\beta(z)} .
$$

By the second equation in (2.1) and (2.21), we have

$$
\begin{aligned}
\Delta_{c} f & =e^{\beta(z)} f(z)+\left(1-e^{\beta(z)}\right) a(z) \\
\Delta_{c}^{2} f & =e^{\beta(z+c)} f(z+c)+\left(1-e^{\beta(z+c)}\right) a(z+c)-\left(e^{\beta(z)} f(z)+\left(1-e^{\beta(z)}\right) a(z)\right) \\
& =e^{\beta(z)} f(z+c)+\left(1-e^{\beta(z)}\right) a(z)-e^{\beta(z)} f(z)-\left(1-e^{\beta(z)}\right) a(z) \\
& =e^{\beta(z)} \Delta_{c} f \\
\Delta_{c}^{3} f & =e^{\beta(z+c)} \Delta_{c} f(z+c)-e^{\beta(z)} \Delta_{c} f=e^{\beta(z)} \Delta_{c}^{2} f=e^{2 \beta(z)} \Delta_{c} f
\end{aligned}
$$

By induction,

$$
\Delta_{c}^{n} f=e^{(n-1) \beta(z)} \Delta_{c} f
$$

Rewriting (2.22), and combining it with the second equation in (2.1), we obtain

$$
\begin{aligned}
\Delta_{c}^{n} f-a(z) & =e^{(n-1) \beta(z)} \Delta_{c} f-a(z) \\
& =e^{(n-1) \beta(z)}\left(e^{\beta(z)}(f(z)-a(z))+a(z)\right)-a(z) \\
& =e^{n \beta(z)}(f(z)-a(z))+a(z)\left(e^{(n-1) \beta(z)}-1\right) .
\end{aligned}
$$

Substituting the first equation in (2.1) into (2.23), we get

$$
\left(e^{\alpha(z)}-e^{n \beta(z)}\right)(f(z)-a(z))=a(z)\left(e^{(n-1) \beta(z)}-1\right) .
$$

If $e^{\alpha(z)}-e^{n \beta(z)} \not \equiv 0,(2.24)$ yields

$$
T(r, f)+S(r, f)=T\left(r,\left(e^{\alpha(z)}-e^{n \beta(z)}\right)(f(z)-a(z))\right)=T\left(r, a(z)\left(e^{(n-1) \beta(z)}-1\right)\right)=S(r, f) .
$$

We get a contradiction again.

Hence, $e^{\alpha(z)}-e^{n \beta(z)} \equiv 0$. By $(2.24)$, we see that $a(z)\left(e^{(n-1) \beta(z)}-1\right) \equiv 0$, which implies $e^{(n-1) \beta(z)} \equiv 1$. That is impossible. 
Step 2. Suppose that $\beta(z)$ is a constant. Now we rewrite the second equation in (2.1) as

$$
\Delta_{c} f=e^{\beta} f(z)+\left(1-e^{\beta}\right) a(z) .
$$

Since $a(z)$ is a periodic function with period $c$, we have

$$
\Delta_{c}^{2} f=e^{\beta} \Delta_{c} f
$$

By induction,

$$
\Delta_{c}^{n} f=e^{(n-1) \beta} \Delta_{c} f .
$$

Then

$$
\Delta_{c}^{n} f-a(z)-e^{(n-1) \beta}\left(\Delta_{c} f-a(z)\right)=a(z)\left(e^{(n-1) \beta}-1\right) .
$$

By Lemma 2.1 and the first equation in (2.1), we deduce that

$$
\frac{a(z)}{f(z)-a(z)}=\frac{\Delta_{c}^{n} f}{f(z)-a(z)}-e^{\alpha(z)}
$$

and

$$
\begin{aligned}
m\left(r, \frac{1}{f(z)-a(z)}\right) & =m\left(r, \frac{1}{a(z)}\left(\frac{\Delta_{c}^{n} f}{f(z)-a(z)}-e^{\alpha(z)}\right)\right) \\
& \leq m\left(r, \frac{\Delta_{c}^{n} f}{f(z)-a(z)}\right)+m\left(r, e^{\alpha(z)}\right)+S(r, f) \\
& =S(r, f) .
\end{aligned}
$$

From (2.27), we have

$$
\begin{aligned}
N\left(r, \frac{1}{f(z)-a(z)}\right) & =T\left(r, \frac{1}{f(z)-a(z)}\right)-m\left(r, \frac{1}{f(z)-a(z)}\right) \\
& =T(r, f(z))+S(r, f) .
\end{aligned}
$$

According to our assumption that $\Delta_{c}^{n} f \not \equiv \Delta_{c} f$ and (2.25), it is easy to see that $e^{(n-1) \beta} \not \equiv 1$. Now suppose that $z_{0}$ is a zero of $f(z)-a(z)$ with multiplicity $\mu$. Since $f(z), \Delta_{c} f$, and $\Delta_{c}^{n} f$ share $a(z) \mathrm{CM}, z_{0}$ is a zero of $\Delta_{c} f-a(z)$ and $\Delta_{c}^{n} f-a(z)$ with multiplicity $\mu$. Therefore, $z_{0}$ is a zero of $\Delta_{c}^{n} f-a(z)-e^{(n-1) \beta}\left(\Delta_{c} f-a(z)\right)$ with multiplicity at least $\mu$. Then by (2.26) and (2.28), we see that

$$
\begin{aligned}
N\left(r, \frac{1}{a(z)\left(e^{(n-1) \beta}-1\right)}\right) & =N\left(r, \frac{1}{\Delta_{c}^{n} f-a(z)-e^{(n-1) \beta}\left(\Delta_{c} f-a(z)\right)}\right) \\
& \geq N\left(r, \frac{1}{f(z)-a(z)}\right) \\
& =T(r, f(z))+S(r, f),
\end{aligned}
$$

which implies $T(r, f(z)) \leq S(r, f)$. That is impossible.

Hence, we must have $\Delta_{c}^{n} f \equiv \Delta_{c} f$, and Theorem 1.1 is proved. 


\section{Proof of Theorem 1.2}

Note that $f(z)$ is a nonconstant entire function of finite order. Since $f(z), \Delta_{c} f$, and $\Delta_{c}^{n} f$ $(n \geq 2)$ share $0 \mathrm{CM}$, we have

$$
\frac{\Delta_{c}^{n} f}{f(z)}=e^{\alpha(z)}, \quad \frac{\Delta_{c} f}{f(z)}=e^{\beta(z)},
$$

where $\alpha(z)$ and $\beta(z)$ are polynomials.

If $\beta(z)$ is a constant, then we can easily get from (3.1)

$$
\Delta_{c}^{n} f=e^{(n-1) \beta} \Delta_{c} f:=C \Delta_{c} f
$$

This completes our proof.

If $\beta(z)$ is not a constant, by assuming that $\frac{\Delta_{c f}^{n} f}{\Delta_{c} f}$ is not a constant, with a similar arguing as in the proof of Theorem 1.1, we can deduce that the case $\operatorname{deg} \beta(z)>1$ is impossible.

For the case $\operatorname{deg} \beta(z)=1$, from (3.1), we can obtain that

$$
f(z+c)=\left(e^{\beta(z)}+1\right) f(z) .
$$

Let $z_{0}$ be a zero of $e^{\beta(z)}+1$. Now we can find that (2.21) still holds here. Then from (2.21), we have $e^{\beta\left(z_{0}+k c\right)}+1=0$, for all $k \in \mathbb{Z}$. Therefore, from (3.2), we see that $z_{k}=z_{0}+k c$ is a zero of $f(z+c)$, then $z_{k+1}=z_{0}+(k+1) c$ is a zero of $f(z)$, for all $k \in \mathbb{Z}$. Suppose that $z_{1}=z_{0}+c$ is a zero of $f(z)$ of order $k_{1}$, then we get from (2.21) and (3.2)

$$
\begin{aligned}
f(z) & =\left(e^{\beta(z-c)}+1\right) f(z-c)=\left(e^{\beta(z)}+1\right)^{2} f(z-2 c)=\cdots \\
& =\left(e^{\beta(z)}+1\right)^{k_{1}+1} f\left(z-\left(k_{1}+1\right) c\right) .
\end{aligned}
$$

This indicates that $z_{1}$ is a zero of $f(z)$ of order at least $k_{1}+1$, which is impossible. Theorem 1.2 is thus proved.

\section{Competing interests}

The authors declare that they have no competing interests.

\section{Authors' contributions}

Both authors drafted the manuscript, and read and approved the final manuscript.

\section{Acknowledgements}

This work was supported by the NNSFC (No. 11301091) and the Guangdong Natural Science Foundation (No. S2013040014347) and the Foundation for Distinguished Young Talents in Higher Education of Guangdong (No. 2013LYM_0037).

Received: 26 July 2014 Accepted: 25 November 2014 Published: 08 Dec 2014

\section{References}

1. Hayman, WK: Meromorphic Functions. Oxford Mathematical Monographs. Clarendon, Oxford (1964)

2. Laine, I: Nevanlinna Theory and Complex Differential Equations. de Gruyter Studies in Mathematics, vol. 15. de Gruyter, Berlin (1993)

3. Yang, CC, Yi, HX: Uniqueness Theory of Meromorphic Functions. Kluwer Academic, Dordrecht (2003)

4. Jank, G, Mues, E, Volkmann, L: Meromorphe funktionen, die mit ihrer ersten und zweiten ableitung einen endlichen wert teilen. Complex Var. Theory Appl. 6(1), 51-71 (1986)

5. Li, P, Yang, CC: Uniqueness theorems on entire functions and their derivatives. J. Math. Anal. Appl. 253(1), 50-57 (2001)

6. Yang, LZ: Further results on entire functions that share one value with their derivatives. J. Math. Anal. Appl. 212, 529-536 (1997)

7. Bergweiler, W, Langley, JK: Zeros of differences of meromorphic functions. Math. Proc. Camb. Philos. Soc. 142 133-147 (2007) 
8. Chen, ZX: Relationships between entire functions and their forward difference. Complex Var. Elliptic Equ. 58(3), 299-307 (2013)

9. Chen, ZX, Shon, KH: Properties of differences of meromorphic functions. Czechoslov. Math. J. 61, 213-224 (2011)

10. Chiang, YM, Feng, SJ: On the Nevanlinna characteristic of $f(z+\eta)$ and difference equations in the complex plane. Ramanujan J. 16(1), 105-129 (2008)

11. Halburd, RG, Korhonen, RJ: Nevanlinna theory for the difference operator. Ann. Acad. Sci. Fenn. Math. 31, 463-478 (2006)

12. Halburd, RG, Korhonen, RJ: Difference analogue of the lemma on the logarithmic derivative with applications to difference equations. J. Math. Anal. Appl. 314(2), 477-487 (2006)

13. Chen, $B Q$, Chen, $Z X, L i, S$ : Uniqueness theorems on entire functions and their difference operators or shifts. Abstr. Appl. Anal. 2012, Article ID 906893 (2012)

14. Heittokangas, J, Korhonen, R, Laine, I, Rieppo, J, Zhang, J: Value sharing results for shifts of meromorphic functions, and sufficient conditions for periodicity. J. Math. Anal. Appl. 355, 352-363 (2009)

15. Zhang, JL: Value distribution and shared sets of differences of meromorphic functions. J. Math. Anal. Appl. 367, 401-408 (2010)

10.1186/1687-1847-2014-311

Cite this article as: Chen and Li: Uniqueness problems on entire functions that share a small function with their difference operators. Advances in Difference Equations 2014, 2014:311

\section{Submit your manuscript to a SpringerOpen ${ }^{\circ}$ journal and benefit from:}

- Convenient online submission

- Rigorous peer review

- Immediate publication on acceptance

- Open access: articles freely available online

- High visibility within the field

- Retaining the copyright to your article 